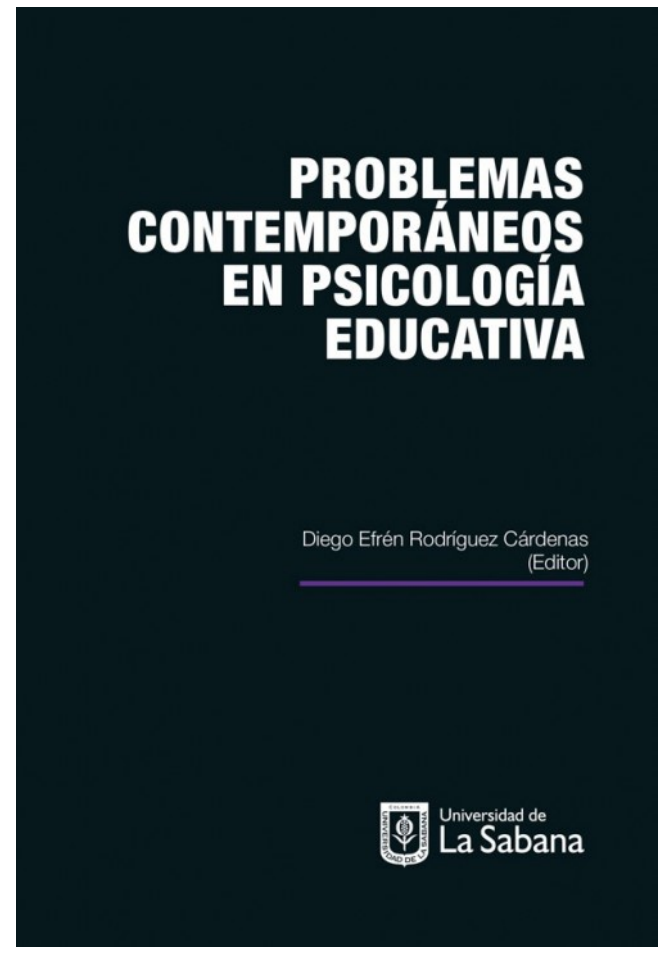

\title{
PROBLEMAS CONTEMPORÁNEOS EN PSICOLOGÍA EDUCATIVA
}

Por: Diego Efrén Rodríguez Cárdenas, Ana María Arias Cardona, Nohemy Marcela Bedoya Ríos, Adriana María Caicedo Tamayo, José David Chávez Vescance, María Fernanda Enríquez Villota, Simón Espinosa Olarte, Fernando Garzón Velásquez, Juliana Gonzales Villa, Martha Rocío González Bernal, María Patricia González Gómez, Diego Fernando Guerrero López, María Inés Menjura Escobar, Jairo Andrés Montes González, Claudia Patricia Ortiz Escobar, Olga Romero Espinosa, lleana Sofía Silva Ordosgoitia, Gloria del Carmen Tobón Vásquez, Laura Paola Valderrama Sánchez, Marcela Valencia Serrano

Formato: Impreso

ISBN: 978-958-12-0449-6

Facultad: Psicología

Colección: Investigación \# 15

DOI: https://doi.org/10.5294/978-958-12-0449-6

Idioma: Español

Precio en dólares: USD $\$ 28,00$

Número de páginas: 334

Palabras clave: Investigación acción, Investigación educativa, Investigación pedagógica, Psicología educativa

\section{$\$ 75.000$}

\section{Reseña del Producto}

Este libro constituye una apuesta de investigadores colombianos de cinco universidades y cinco departamentos diferentes (Antioquia, Caldas, Cundinamarca, Nariño y Valle del Cauca), vinculados al nodo de psicología educativa de la Red de Investigadores de la Asociación Colombiana de Facultades de Psicología (Ascofapsi), organizados para discutir coordenadas conceptuales para la comprensión de esta importante disciplina, pero también para evidenciar tópicos, problemas y aproximaciones metodológicas relevantes para la investigación en el área y la producción de conocimiento pertinente en una diversidad de contextos educativos. El libro tiene el valor de evidenciar el papel de la psicología educativa, no solo en los contextos escolares tradicionales, sino también en contextos extraescolares, aspecto de primer orden en la agenda de esta disciplina. Por otra parte, tiene el valor de mostrar diferentes aproximaciones metodológicas para la comprensión del objeto de estudio. El abordaje teórico, la pertinencia de los temas, la apertura hacia contextos escolares y no escolares y la diversidad de aproximaciones metodológicas constituyen su riqueza fundamental.

\section{Información Adicional}

Peso: $1 \mathrm{~kg}$

Tamaño: $17 \times 24 \times 2 \mathrm{~cm}$

Sku: 9789581204496

Código topografico: 370.15

\section{Tabla de contenido}


I. TEORÍA PSICOEDUCATIVA

1. Psicología y educación: teóricos relevantes y sus aportes

2. Psicología, cultura y educación

II. INVESTIGACIÓN EN CONTEXTOS EDUCATIVOS ESCOLARES

3. Estilos cognitivos y educación en la diversidad: discusión de resultados de investigación

4. Relación entre la secuencia numérica convencional en lengua de señas colombiana y la comprensión numérica en niños sordos

5. Análisis cognitivo de tarea para la investigación en el uso educativo de las tecnologías de la información y de la comunicación

6. La inclusión educativa de niños con discapacidad desde la mirada de los profesores y los compañeros

III. INVESTIGACIÓN EN CONTEXTOS EDUCATIVOS NO ESCOLARES

7. Proyectos educativos más allá de la escuela: sistematización de la experiencia "Área educada: educación con calidad para la sostenibilidad"

8. Evaluación de los beneficios del Proyecto de Educación a Campo Abierto: un análisis a partir del desarrollo juvenil positivo 